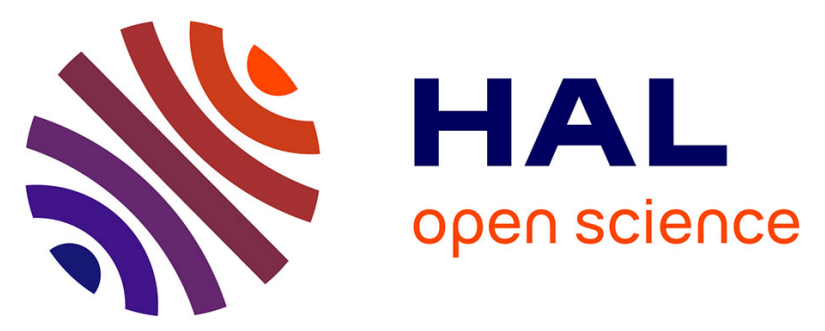

\title{
MALDI-TOF mass spectrometry-based identification of Listeria species in surveillance: a prospective study
}

Pierre Thouvenot, Guillaume Vales, Hélène Bracq-Dieye, Nathalie

Tessaud-Rita, Mylène M Maury, Alexandra Moura, Marc Lecuit, Alexandre Leclercq

\section{To cite this version:}

Pierre Thouvenot, Guillaume Vales, Hélène Bracq-Dieye, Nathalie Tessaud-Rita, Mylène M Maury, et al.. MALDI-TOF mass spectrometry-based identification of Listeria species in surveillance: a prospective study. Journal of Microbiological Methods, 2017, 144, pp.29-32. 10.1016/j.mimet.2017.10.009 . pasteur-02320046

\section{HAL Id: pasteur-02320046 \\ https://hal-pasteur.archives-ouvertes.fr/pasteur-02320046}

Submitted on 18 Oct 2019

HAL is a multi-disciplinary open access archive for the deposit and dissemination of scientific research documents, whether they are published or not. The documents may come from teaching and research institutions in France or abroad, or from public or private research centers.
L'archive ouverte pluridisciplinaire HAL, est destinée au dépôt et à la diffusion de documents scientifiques de niveau recherche, publiés ou non, émanant des établissements d'enseignement et de recherche français ou étrangers, des laboratoires publics ou privés.

\section{(ㅇ)(1) $\$$}

Distributed under a Creative Commons Attribution - NonCommercial - NoDerivatives 44.0 


\section{MALDI-TOF mass spectrometry-based identification of Listeria species in surveillance: a prospective study}

Pierre Thouvenot ${ }^{\mathrm{a}}$, Guillaume Vales ${ }^{\mathrm{a}}$, Hélène Bracq-Dieye ${ }^{\mathrm{a}}$, Nathalie Tessaud-Rita ${ }^{\mathrm{a}}$, Alexandra Moura ${ }^{\mathrm{a}}$, Mylène M. Maury ${ }^{\mathrm{a}}$, Marc Lecuit ${ }^{\mathrm{a}, \mathrm{b}, \mathrm{c}}$,\# and Alexandre Leclercq ${ }^{\mathrm{a}}$,\#

${ }^{a}$ Institut Pasteur, Biology of Infection Unit, National Reference Centre and WHO Collaborating Centre for Listeria, Paris, France

${ }^{\mathrm{b}}$ Inserm U1117, Paris, France

c Paris Descartes University, Necker-Enfants Malades University Hospital, Department of Infectious Diseases and Tropical Medicine, Institut Imagine, APHP, Paris, France

\# Corresponding authors: E-mail addresses: Institut Pasteur, Biology of Infection Unit, National Reference Centre and WHO Collaborating Centre for Listeria, 28 rue du Docteur Roux, F-75724 Paris cedex 15, France. Phone: 3301406131 12. Fax: 3301406135 67. E-mails: marc.lecuit@pasteur.fr, alexandre.leclercq@pasteur.fr 


\section{ABSTRACT}

This study aimed to evaluate MALDI-TOF MS for species discrimination of Listeria in the context of routine surveillance. MALDI-TOF MS yielded 100\% accuracy for the identification of

L. monocytogenes, L. innocua, L. ivanovii, L. fleischmannii, L. grayi, L. seeligeri, $L$. weihenstephanensis and L. welshimeri, as confirmed by whole genome analyses. 


\section{Highlights}

- MALDI-TOF MS correctly identifies human pathogenic Listeria species.

- Prospective collected isolates $(n=1,201)$ were identified with $100 \%$ of accuracy.

- Reliable identification is linked to a good quality reference library of MALDI-TOF MS spectra.

- MALDI-TOF MS is a fast and cost-effective tool for routine Listeria identification.

\section{Keywords}

Listeria identification, MALDI-TOF, mass spectrometry, food, public health surveillance. 
Listeria monocytogenes is a Gram-positive bacillus and the cause of listeriosis, a severe human foodborne infection (Vazquez-Boland et al., 2001). It belongs to the genus Listeria, which currently includes 17 species: L. aquatica, L. booriae, L. cornellensis, L. fleischmannii, L. floridensis, L. grandensis, L. grayi, L. innocua, L. ivanovii, L. marthii, L. monocytogenes, $L$. newyorkensis, L. riparia, L. rocourtiae, L. seeligeri, L. weihenstephanensis and L. welshimeri (Orsi and Wiedmann, 2016). Of these, five are routinely isolated from food: L. monocytogenes, $L$. innocua, L. ivanovii, L. seeligeri, and L. welshimeri, but only L. monocytogenes, and rarely $L$. ivanovii, are of medical concern (Guillet et al., 2010). In the context of Listeria surveillance, it is therefore critical to use an accurate, simple, rapid and cost-effective method to distinguish between these species. Current identification tools, such as API-Listeria (bioMérieux, Marcy l'Etoile, France) (Bille et al., 1992), PCR serogrouping (Doumith et al., 2004) and 16S rRNA gene sequencing (Sontakke et al., 2009) are expensive and time-consuming.

Matrix-Assisted Laser Desorption/Ionization-Time of Flight Mass Spectrometry (MALDI-TOF MS) has become a reliable tool for bacterial identification in clinical microbiology (Angeletti, 2016). The identification accuracy, low cost of reagents/consumables per sample after purchasing and maintenance of equipment, and speed of data acquisition are the most prominent advantages of this technology. MALDI-TOF MS has been successfully used for the detection of $L$. monocytogenes from selective enrichment broth (Jadhav et al., 2014), rapid identification of Listeria species (Barbuddhe et al., 2008; Rychert et al., 2013; Hsueh et al., 2014; Jadhav et al., 2015; Ojima-Kato et al., 2016), discrimination of lineages (Ojima-Kato et al., 2016) and subtyping of Listeria monocytogenes (Barbuddhe et al., 2008; Jadhav et al., 2015; Ojima-Kato et al., 2016). Previous studies used the Andromas MALDI-TOF MS system (Paris, France) (Farfour et al., 2012), the Shimadzu Corporation MALDI-TOF MS system (Kyoto, Japan) (Jadhav et al., 2014, 2015; Ojima-Kato et al., 2016) or the Vitek MALDI-TOF MS system (bioMérieux, Marcy 
l'Etoile, France) (Rychert et al., 2013), and did not exhibit high species identification accuracy. The Bruker Daltonics (Bremen, Germany) MALDI-TOF MS system has been used with success, but on a limited number of phenotypically identified strains and to establish mass spectral fingerprints of some reference strains (Barbuddheet al., 2008; Hsueh et al., 2014).

In the context of a National Reference Centre for Listeria, that receives around 1,800 Listeria isolates per year from clinical (20\%) and food (80\%) origins, we evaluated the Bruker Daltonics system of MALDI-TOF MS Biotyper as a method for rapid identification of L. monocytogenes and the 16 other Listeria spp. described until now. We also compared the results to phenotypicbased identification. We first performed a validation study, and then used this system prospectively in the context of Listeria surveillance in France. Its accuracy was confirmed by bacterial identification using average nucleotide identity BLAST (ANIb) determined from whole genome sequences (Goris et al., 2007).

A validation set of 386 Listeria strains representative of Listeria genus and L. monocytogenes diversity (Chenal-Francisque et al., 2015) was first analyzed (Table 1). A particular focus was given to L. monocytogenes ( $n=353$ ), to fully cover the diversity of this species, which is the main common pathogenic Listeria isolated in clinical and food microbiological laboratories and responsible for human listeriosis. In addition, a set of 34 non-Listeria isolates corresponding to frequent contaminants often phenotypically misidentified as Listeria were used as negative controls (Table 2). A prospective analysis was then carried out for all the Listeria strains received at the French National Reference Center of Listeria (NRCL) in 2016 ( $n=1,201$, Table 3).

Strains were grown overnight (18h) on Brain Heart Infusion (BHI; Becton Dickinson Difco, Franklin Lakes, USA) agar plates at $35^{\circ} \mathrm{C}$. The samples were prepared by full protein extraction and analysed according to the Bruker Daltonics instructions. As described in previous studies (Barbuddhe et al., 2008; Hsueh et al., 2014; Capocefalo et al., 2016,), this full protein extraction 
was crucial to obtain reliable Listeria identification to the species level (data not shown). Briefly, colonies were transferred into a 2-ml screw-cap extraction tube (Eppendorf, Germany) containing $300 \mu \mathrm{l}$ of distilled water and then mixed with $900 \mu \mathrm{l}$ of absolute ethanol by pipetting. The suspension was pelleted by centrifugation at $13,000 \mathrm{rpm}$ for $2 \mathrm{~min}$, air dried, and then reconstituted with $50 \mu \mathrm{l}$ of $70 \%$ formic acid (Sigma-Adlrich, Darmstadt, Germany) before the addition of $50 \mu \mathrm{l}$ of acetonitrile (Sigma-Aldrich) to the mixture (Bizzini et al., 2010). The mixture was then centrifuged at 13,000 rpm for $2 \mathrm{~min}$. Next, $1 \mu \mathrm{L}$ of the supernatant was deposited in duplicate spots onto a polished steel MALDI-TOF MS target plates (Bruker Daltonics, Bremen, Germany) and air dried at room temperature. As quality control system, $1 \mu \mathrm{L}$ of Bruker Bacterial Test Standard (BTS; Bruker Daltonics) was also used at the beginning and end of every series of samples. Dried spots were overlaid with $1 \mu 1$ of a HCCA matrix (a saturated solution of $\alpha$-cyano4-hydroxycinnamic acid (HCCA) in acetonitrile 50\%, water 47.5\% and trifluoroacetic acid 2.5\%; Bruker Daltonics) and let air dry again at room temperature according to manufacturer's instructions (Bruker Daltonics). The MALDI-plate was inserted into the source of a microFlex LT/SH MALDI-TOF MS instrument (Bruker Daltonics) with a 60-Hz nitrogen laser (337 nm wavelength) and automatically read. The spectra were collected in the linear positive mode in a mass range covering m/z 1,960-20,132 in the FlexControl v3.4 software (Bruker Daltonics). All spectra ranging from the mass-to-charge ratio $(\mathrm{m} / \mathrm{z}) 2,000-20,000$, corresponding to the resulting ribosomal protein profiles, were analyzed using the MALDI BioTyper (MBT) compass explorer software v4.1.60 (Bruker Daltonics) and compared to reference spectra of the MALDI BioTyper Reference library (database [DB-5989] MS; Bruker Daltonics) for automatic identification. The whole treatment from processing acquired spectra to identification was performed automatically without user intervention using the integrated pattern matching algorithm of the software (Barbuddhe et al., 2008). Scores of $\geq 2.000$ indicated species-level identification, scores of 1.700 - 
1.999 indicated genus-level identification, and scores of $<1.699$ indicated no reliable identification. For each isolate, 2 spots were submitted to two analyses to obtain at least 4 identical and reliable scores results $(2.0 \leq$ score $\leq 3.0)$. Automatized bacterial identification were valid if the BTS was identified as Escherichia coli DH5-alpha. All isolates with discrepant identification results between the molecular and Bruker Biotyper methods were tested twice.

Conventional identification was carried out by API-Listeria (bioMérieux) combined with hemolysis test on horse blood agar according to manufacturer's instructions (Bille et al., 1992). PCR serogrouping of Listeria isolates was determined by a multiplex PCR and interpreted as described by Doumith et al. (2004) and Leclercq et al. (2011). Genome sequences were obtained after DNA extraction (DNeasy Blood \& Tissue kit, Qiagen, Denmark), library preparation (Nextera XT DNA Sample kit, Illumina, California, USA) and sequencing using NextSeq 500 (2 x $150 \mathrm{bp)}$ platform (Illumina, California, USA), according to the manufacturer's protocol. Sequence assemblies were built using CLC Assembly Cell 4.3.0. (Qiagen, Hilden, Germany) from FqCleaner filtered reads with an average read coverage higher than $40 \mathrm{X}$, as described previously (Moura et al., 2017). Genome-based species identification was determined by average nucleotide identity BLAST (ANIb) analysis against the Listeria species reference genomes deposited at NCBI database (https://www.ncbi.nlm.nih.gov), as described previously (Goris et al., 2007). The congruence between MALDI identification results and those obtained by classical reference methods and whole genome sequencing was evaluated.

The accuracy of MALDI-TOF identification results is shown in Table 1. Unambiguous identification at the species level was obtained for the 8 most frequently isolated species: $L$. monocytogenes (353/353), L. welshimeri (3/3), L. innocua (4/4), L. ivanovii (4/4), L. fleischmannii (3/3), L. grayi (2/2), L. seeligeri (4/4), and L. weihenstephanensis (1/1). For strains with subspecies variants (L. ivanovii, L. fleischmannii and L. grayi), MALDI-TOF MS provided a 
correct species identification, but not at the sub-species level. As expected, since the corresponding reference profiles are absent from the current version of MALDI Biotyper reference library, unreliable identification was obtained for $L$. grandensis, L. rocourtiae, $L$. aquatica, L. cornellensis, L. riparia, L. floridensis, L. booriae and L. newyorkensis. These last species could not be identified by API-Listeria. Of note, these species have never been isolated in the context of Listeria surveillance in France. Finally, L. marthii, a rarely isolated species, was always misidentified as L. monocytogenes with acceptable scores, also due to the lack of reference profile for this species in the database. None of the negative controls were misidentified as Listeria species (Table 2).

We then applied this method for the prospective identification of 1,201 strains received in the context of Listeria national surveillance in France from April 2016 to December 2016 (Table 3). These strains were isolated from human $(n=299)$, food $(n=731)$, environmental $(n=155)$, animal $(n=2)$ or unknown origin from owncheck food manufacturers $(n=14)$ samples. In total, 5 species were correctly identified at the species level and included L. monocytogenes $(\mathrm{n}=1,112), L$. innocua $(\mathrm{n}=65)$, L. welshimeri $(\mathrm{n}=20)$, L. seeligeri $(\mathrm{n}=3)$ and L. ivanovii $(\mathrm{n}=1)$ isolates. All isolates were correctly identified by MALDI-TOF MS at the species level, as confirmed by ANIb identification from whole genome sequence analysis.

This work demonstrates the complete reliability of MALDI-TOF mass spectrometry as a rapid (less than 40 minutes) approach for the identification of Listeria in human, animal, food, and environmental microbiology, with $100 \%$ of accuracy for L. monocytogenes, L. innocua, L. ivanovii, L. fleischmannii, L. grayi, L. seeligeri, L. weihenstephanensis and L. welshimeri identification. This study also underlines the importance of a good quality and continuously updated reference MBT library for accurate identification of Listeria. 


\section{ACKNOWLEDGMENTS}

We thank Dr. Martin Wiedmann (Cornell University, USA) for providing type strains of $L$. marthii, L. grandensis, L. aquatica, L. cornellensis, L. riparia and L. floridensis, Dr. Leo Meile (Swiss Federal Institute of Technology Zurich, Switzerland) for L. fleischmannii, Dr. C. Bizet (Collection de l'Institut Pasteur, France) for providing type strains of L. weihenstephanensis, $L$. booriae, L. newyorkensis and the access to the Bruker Daltonics MALDI-TOF MS system. We are also thankful to the P2M platform (Institut Pasteur, Paris, France) for genome sequencing. This work was supported by Institut Pasteur and Public Health France.

Conflict of interest: none 


\section{REFERENCES}

Angeletti, S., 2016. Matrix assisted laser desorption time of flight mass spectrometry (MALDITOF MS) in clinical microbiology. J. Microbiol. Methods.

Barbuddhe, S.B., Maier, T., Schwarz, G., Kostrzewa, M., Hof, H., Domann, E., Chakraborty, T., Hain, T., 2008. Rapid identification and typing of Listeria species by matrix-assisted laser desorption ionization-time of flight mass spectrometry. Appl. Environ. Microbiol. 74, $5402-5407$.

Bille, J., Catimel, B., Bannerman, E., Jacquet, C., Yersin, M.N., Caniaux, I., Monget, D., Rocourt, J., 1992. API Listeria, a new and promising one-day system to identify Listeria isolates. Appl. Environ. Microbiol. 58, 1857-1860.

Bizzini, A., Durussel, C., Bille, J., Greub, G., Prod'hom, G., 2010. Performance of matrixassisted laser desorption ionization-time of flight mass spectrometry for identification of bacterial strains routinely isolated in a clinical microbiology laboratory. J. Clin. Microbiol. $48,1549-1554$.

Capocefalo, M., Ridley, E.V., Tranfield, E.Y., Thompson, K.C., 2016. MALDI-TOF MS: a rapid microbiological confirmation technique for food and water analysis. In: N. Cook, M. D'Agostino, K. C. Thompson (Eds.), Molecular microbial diagnostic methods: Pathways to implementation for the food and water industries, Elsevier, Academic Press, London, UK, pp. $185-220$.

Chenal-Francisque, V., Maury, M.M., Lavina, M., Touchon, M., Leclercq, A., Lecuit, M., Brisse, S., 2015. Clonogrouping, a Rapid Multiplex PCR Method for Identification of Major Clones of Listeria monocytogenes. J. Clin. Microbiol. 53, 3355-3358. 
Doumith, M., Buchrieser, C., Glaser, P., Jacquet, C., Martin, P., 2004. Differentiation of the major Listeria monocytogenes serovars by multiplex PCR. J. Clin. Microbiol. 42, 38193822.

Farfour, E., Leto, J., Barritault, M., Barberis, C., Meyer, J., Dauphin, B., Le Guern, A.S., Lefleche, A., Badell, E., Guiso, N., Leclercq, A., Le Monnier, A., Lecuit, M., RodriguezNava, V., Bergeron, E., Raymond, J., Vimont, S., Bille, E., Carbonnelle, E., Guet-Revillet, H., Lecuyer, H., Beretti, J.L., Vay, C., Berche, P., Ferroni, A., Nassif, X., Join-Lambert, O., 2012. Evaluation of the Andromas matrix-assisted laser desorption ionization-time of flight mass spectrometry system for identification of aerobically growing Gram-positive bacilli. J. Clin. Microbiol. 50, 2702-2707.

Goris, J., Konstantinidis, K.T., Klappenbach, J.A., Coenye, T., Vandamme, P., Tiedje, J.M., 2007. DNA-DNA hybridization values and their relationship to whole-genome sequence similarities. Int. J. Syst. Evol. Microbiol. 57, 81-91.

Guillet, C., Join-Lambert, O., Le Monnier, A., Leclercq, A., Mechai, F., Mamzer-Bruneel, M.F., Bielecka, M.K., Scortti, M., Disson, O., Berche, P., Vazquez-Boland, J., Lortholary, O., Lecuit, M., 2010. Human listeriosis caused by Listeria ivanovii. Emerg. Infect. Dis. 16, $136-138$.

Hsueh, P.R., Lee, T.F., Du, S.H., Teng, S.H., Liao, C.H., Sheng, W.H., Teng, L.J., 2014. Bruker biotyper matrix-assisted laser desorption ionization-time of flight mass spectrometry system for identification of Nocardia, Rhodococcus, Kocuria, Gordonia, Tsukamurella, and Listeria species. J. Clin. Microbiol. 52, 2371-2379.

Jadhav, S., Sevior, D., Bhave, M., Palombo, E.A., 2014. Detection of Listeria monocytogenes from selective enrichment broth using MALDI-TOF Mass Spectrometry. J. Proteomics. 97, 100-106. 
Jadhav, S., Gulati, V., Fox, E.M., Karpe, A., Beale, D.J., Sevior, D., Bhave, M., Palombo, E.A., 2015. Rapid identification and source-tracking of Listeria monocytogenes using MALDITOF mass spectrometry. Int. J. Food Microbiol. 202, 1-9.

Leclercq, A., Chenal-Francisque, V., Dieye, H., Cantinelli, T., Drali, R., Brisse, S., Lecuit, M., 2011. Characterization of the novel Listeria monocytogenes PCR serogroupingprofile IVb-v1. Int. J. Food Microbiol. 147(1), 74-7.

Moura, A., Tourdjman, M., Leclercq, A., Hamelin, E., Laurent, E., Fredriksen, N., Van Cauteren, D., Bracq-Dieye, H., Thouvenot, P., Vales, G., Tessaud-Rita, N., Maury, M.M., Alexandru, A., Criscuolo, A., Quevillon, E., Donguy, M.P., Enouf, V., de Valk, H., Brisse, S., Lecuit, M., 2017. Real-time whole-genome sequencing for surveillance of Listeria monocytogenes, France. Emerg Infect Dis. 23(9):1462-1470.

Ojima-Kato, T., Yamamoto, N., Takahashi, H., Tamura, H., 2016. Matrix-assisted Laser Desorption Ionization-Time of Flight Mass Spectrometry (MALDI-TOF MS) Can Precisely Discriminate the Lineages of Listeria monocytogenes and Species of Listeria. PLoS One. 11, e0159730.

Orsi, R.H., Wiedmann, M., 2016. Characteristics and distribution of Listeria spp., including Listeria species newly described since 2009. Appl. Microbiol. Biotechnol. 100, 5273-5287. Rychert, J., Burnham, C.A., Bythrow, M., Garner, O.B., Ginocchio, C.C., Jennemann, R., Lewinski, M.A., Manji, R., Mochon, A.B., Procop, G.W., Richter, S.S., Sercia, L., Westblade, L.F., Ferraro, M.J., Branda, J.A., 2013. Multicenter evaluation of the Vitek MS matrix-assisted laser desorption ionization-time of flight mass spectrometry system for identification of Gram-positive aerobic bacteria. J. Clin. Microbiol. 51, 2225-2231.

Sontakke, S., Cadenas, M.B., Maggi, R.G., Diniz, P.P., Breitschwerdt, E.B., 2009. Use of broad range16S rDNA PCR in clinical microbiology. J. Microbiol. Methods. 76, 217-225. 
Vazquez-Boland, J.A., Kuhn, M., Berche, P., Chakraborty, T., Dominguez-Bernal, G., Goebel, W., Gonzalez-Zorn, B., Wehland, J., Kreft, J., 2001. Listeria pathogenesis and molecular virulence determinants. Clin. Microbiol. Rev. 14, 584-640. 


\section{Table 1}

Results of species and subspecies identification by applying the MALDI-TOF MS method on a validation set of 386 Listeria isolates ${ }^{\text {a }}$.

\begin{tabular}{|c|c|c|c|}
\hline Species/subspecies & PCR serogroups ${ }^{b}$ & Hemolysis & $\begin{array}{l}\text { Correct } \\
\text { MALDI-TOF } \\
\text { identification }(\%)\end{array}$ \\
\hline L. monocytogenes & IIa, IIb, IIc, IVb, IVb-v1, L & + & $353 / 353(100)$ \\
\hline L. innocua & $\mathrm{L}$ & - & 4/4 (100) \\
\hline L. ivanovii & $\mathrm{L}$ & + & 4/4 (100) \\
\hline L. ivanovii subsp. ivanovii & $\mathrm{L}$ & + & $3 / 3(100)$ \\
\hline L. ivanovii subsp. londoniensis & $\mathrm{L}$ & + & $1 / 1(100)$ \\
\hline L. welshimeri & $\mathrm{L}$ & - & $3 / 3(100)$ \\
\hline L. seeligeri & $\mathrm{L}$ & + & 4/4 (100) \\
\hline L. grayi & $\mathrm{L}$ & - & $2 / 2(100)$ \\
\hline L. gayi subsp. grayi & $\mathrm{L}$ & - & $1 / 1(100)$ \\
\hline L. grayi subsp. murrayi & $\mathrm{L}$ & - & $1 / 1(100)$ \\
\hline L. fleischmannii & $\mathrm{L}$ & - & $3 / 3(100)$ \\
\hline L. fleischmannii subsp. coloradensis & $\mathrm{L}$ & - & $1 / 1(100)$ \\
\hline L. fleischmannii subsp. fleischmannii & $\mathrm{L}$ & - & $2 / 2(100)$ \\
\hline L. weihenstephanensis & / & - & $1 / 1(100)$ \\
\hline L. marthii & $\mathrm{L}$ & - & $0 / 4(0)^{\mathrm{c}}$ \\
\hline L. aquatica & $\mathrm{L}$ & - & $0 / 1(0)^{\mathrm{d}}$ \\
\hline L. booriae & / & - & $0 / 1(0)^{\mathrm{d}}$ \\
\hline L. cornellensis & / & - & $0 / 1(0)^{\mathrm{d}}$ \\
\hline L. floridensis & $\mathrm{L}$ & - & $0 / 1(0)^{\mathrm{d}}$ \\
\hline L. grandensis & / & - & $0 / 1(0)^{\mathrm{d}}$ \\
\hline L. newyorkensis & / & - & $0 / 1(0)^{\mathrm{d}}$ \\
\hline L. riparia & / & - & $0 / 1(0)^{\mathrm{d}}$ \\
\hline L. rocourtiae & I & - & $0 / 1(0)^{\mathrm{d}}$ \\
\hline
\end{tabular}

${ }^{a}$ Isolates were selected to represent the diversity of Listeria species. The second and third columns indicate, for each species, the PCR serogroups and the hemolysis results on horse blood 
agar plates (bioMérieux), respectively. Proportions of correctly identified isolates by MALDITOF MS are given in the fourth column relatively to API-Listeria identification and genomebased identification results by average nucleotide identity BLAST (ANIb).

${ }^{\mathrm{b}}$ according to Doumith et al. (2004) and Leclercq et al. (2011). PCR serogroup L comprised strains of serovars $4 \mathrm{a}, 4 \mathrm{ab}, 4 \mathrm{c}$ of $L$. monocytogenes and other species, except $L$. booriae, $L$. cornellensis, L. grandensis, L. newyorkensis, L. riparia, L. rocourtiae, and L. weihenstephanensis.

${ }^{\mathrm{c}}$ Misidentified species

${ }^{\mathrm{d}}$ Non-identified species or low scores of identification (scores $\leq 1.99$ ) 


\section{TABLE 2.}

Results of species identification by applying the MALDI-TOF MS method on a negative controls set of 34 non Listeria isolates.

\begin{tabular}{lll}
\hline Species & $\begin{array}{l}\text { MALDI-TOF } \\
\text { score }\end{array}$ & $\begin{array}{l}\text { Correct MALDI-TOF } \\
\text { identification }{ }^{\text {a }}\end{array}$ \\
\hline Achromobacter xylosoxidans & 2.148 & $1 / 1(100 \%)$ \\
Bacillus cereus & $1.86-2.13$ & $3 / 3(100 \%)$ \\
Bacillus circulans & 1.91 & $1 / 1(100 \%)$ \\
Bacillus pumilus & 1.85 & $1 / 1(100 \%)$ \\
Carnobacterium maltaromaticum & 2.15 & $1 / 1(100 \%)$ \\
Enterococcus faecalis & $2.22-2.62$ & $17 / 17(100 \%)$ \\
Erysipelothrix rhusiopathiae & 2.18 & $1 / 1(100 \%)$ \\
Microbacterium arborescens & 2.22 & $1 / 1(100 \%)$ \\
Staphylococcus epidermidis & $1.81-2.04$ & $7 / 7(100 \%)$ \\
Staphylocccus warneri & 2.1 & $1 / 1(100 \%)$ \\
\hline compared with genome-based & &
\end{tabular}

${ }^{a}$ compared with genome-based species identification using the average nucleotide identity BLAST (ANIb) analyses 


\section{Table 3.}

Results of species identification by applying the MALDI-TOF MS method on a validation set of 1201 Listeria isolates.

\begin{tabular}{llll}
\hline Species & PCR serogroups & & \\
& & Hemolysis & $\begin{array}{l}\text { Correct MALDI-TOF } \\
\text { MS identification }\end{array}$ \\
\hline L. innocua & $\mathrm{L}$ & - & $65 / 65(100 \%)$ \\
L. ivanovii & $\mathrm{L}$ & + & $1 / 1(100 \%)$ \\
L. monocytogenes & $\mathrm{IIa}, \mathrm{IIb}, \mathrm{IIc}, \mathrm{IVb}, \mathrm{IVb}-\mathrm{v} 1$ & + & $1112 / 1112(100 \%)$ \\
L. seeligeri & $\mathrm{L}$ & + & $3 / 3(100 \%)$ \\
L. welshimeri & $\mathrm{L}$ & - & $20 / 20(100 \%)$ \\
\hline
\end{tabular}

${ }^{\text {a }}$ according to Doumith et al. (2004) and Leclercq et al. (2011). PCR serogroup L comprised strains of serovars $4 \mathrm{a}, 4 \mathrm{ab}, 4 \mathrm{c}$ of $L$. monocytogenes and other species, except $L$. booriae, $L$. cornellensis, L. grandensis, L. newyorkensis, L. riparia, L. rocourtiae, and L. weihenstephanensis.

${ }^{\mathrm{b}}$ Compared with genome-based species identification using the average nucleotide identity BLAST (ANIb) analyses. 